\title{
POBREZA: PERCEPCIÓN Y ENFOQUES. UN ANÁLISIS PARA VENEZUELA ${ }^{1}$
}

\author{
Omaira Peña ${ }^{2}$ \\ Pedro Harmath ${ }^{3}$ \\ Rafael Acevedo ${ }^{4}$ \\ Universidad Centroccidental Lisandro Alvarado (UCLA), Venezuela
}

http://dx.doi.org/10.5209/rev_NOMA.2013.42348

\begin{abstract}
Resumen.- Este artículo aborda el fenómeno de la pobreza bajo un enfoque multimétodo. Para ello, en principio se presenta un arqueo teórico basado en la revisión de algunos trabajos realizados por ciertos estudiosos, que han incursionado acerca del flagelo objeto de análisis en algunos países incluyendo a Venezuela; para así, abrir paso a un análisis más profundo de los hallazgos encontrados en el trabajo de Harmath y Acevedo (2009). La discusión generada en este trabajo, en términos globales refleja que los rasgos de pobreza en el país, se deben principalmente a la falta de distribución equitativa de los recursos generados por la renta petrolera a lo largo del tiempo; así como a la no concepción óptima de estrategias sociales que canalicen el problema objeto de estudio, desde un punto de vista más "humano-cultural" con el propósito de que la lucha contra él sea más profunda y arraigada.
\end{abstract}

Palabras clave.- enfoque multimétodo, pobreza total, Venezuela

\section{Poverty: perceptions and approaches. An analysis for Venezuela}

Abstract.- This article discusses the phenomenon of poverty under a multi-method approach. This in principle provides a theoretical tonnage based on the revision of some works by some scholars, who have ventured on the scourge analyzed in some countries including Venezuela, to well, make way for a deeper analysis of the findings from the work of Harmath and Acevedo (2009). The discussion generated in this study, overall reflects the features of poverty in the country, are mainly due to the lack of equitable distribution of resources generated by oil revenues over time, as well as to non-optimal design social strategies to channel the problem under study, from a point of view more "human-cultural" for the purpose of fighting him more deep rooted.

Keywords.- multi-method approach, total poverty, Venezuela

Códigos JEL: I32, 138

\footnotetext{
${ }^{1}$ El presente artículo es un producto del Proyecto de Investigación "Pobreza en Venezuela: Un enfoque multimétodo" inscrito en el CDCHT-UCLA bajo el Código 010-RCT-2011 por parte de los integrantes del "Grupo Economía". Dicho proyecto se encuentra adscrito al Departamento de Estudios Básicos y Sociales del Decanato de Ciencias y Tecnología de la Universidad Centroccidental Lisandro Alvarado (UCLA). Los autores declaran que el orden de aparición de sus nombres no indica jerarquía entre ellos en cuanto al trabajo realizado, ya que, todos admiten y aceptan tener la misma proporción de autoría.

${ }^{2}$ Profesora Titular del Departamento de Estudios Básicos y Sociales del Decanato de Ciencias y Tecnología de la Universidad Centroccidental Lisandro Alvarado. Msc. en Administración del Trabajo y Relaciones Laborales (Universidad de Carabobo, Venezuela) y Estudiante del Doctorado en Estudios del Desarrollo (Universidad Central de Venezuela). E-mail: opena@ucla.edu.ve

${ }^{3}$ Profesor Asistente del Departamento de Investigación de Operaciones y Estadística del Decanato de Ciencias y Tecnología de la Universidad Centroccidental Lisandro Alvarado. Msc. en Economía (Universidad de Los Andes, Venezuela) y Estudiante del Doctorado en Matemática (UCLA, Venezuela).

E-mail: pedro.harmath@ucla.edu.ve

${ }^{4}$ Profesor Asistente del Departamento de Estudios Básicos y Sociales del Decanato de Ciencias y Tecnología de la Universidad Centroccidental Lisandro Alvarado. Msc. en Economía (Universidad de Los Andes, Venezuela) y Doctor en Gerencia (Universidad Yacambú, Venezuela). E-mail: rafaelacevedorueda@yahoo.es
} 


\section{Introducción}

Según Harmath y Acevedo (2009), "La pobreza es una característica común, y muy arraigada, principalmente en los países subdesarrollados y por ello, es objeto de estudio de muchas investigaciones por el trasfondo social y humano que encierra" (p.162). Su presencia se observa en aquellas naciones que están atravesando una etapa de crecimiento y desarrollo económico, siendo una de sus causas las olas migratorias en busca de mejores condiciones de vida, al igual que en países desarrollados, impulsado por la necesidad de contratar mano de obra barata, entre otros aspectos.

Este fenómeno o flagelo social, se vincula a elementos omnipresentes en todas las sociedades contemporáneas, entre los que destacan: (i) la ausencia de bienestar material en grandes sectores de la población alejados de la posibilidad de alcanzar oportunidades para desarrollar sus capacidades como seres humanos integrantes de un sistema social; (ii) la disminución extrema de la producción y el consumo de alimentos que afecta la estabilidad de la vida individual y la vida social; (iii) la creciente dificultad para acceder a los servicios básicos de salud, educación y hábitat de calidad y (iv) la pérdida de valores y cultura productiva entre sectores de la población que impide a éstos transformarse en ciudadanos y agentes productivos.

Cabe destacar que existen innumerables acepciones del término pobreza. El Instituto Nacional de Estadística de Venezuela (INE), en su página web, indica que un hogar se encuentra en condiciones de pobreza, si sus ingresos percibidos no logran cubrir el costo de la canasta normativa de consumo total, la cual incluye alimentos y otros bienes y servicios. En otras investigaciones, los autores (entre ellos, Puente y otros, 2005; Gontero y otros, 2005) se inclinan a definirla como la circunstancia económica en la que una persona carece de ingresos suficientes para acceder a los niveles mínimos de atención médica, alimentos, vivienda, ropa, educación entre otros aspectos (las llamadas necesidades básicas insatisfechas NBI).

Amartya Sen, citado por Molina (2009), la define desde la perspectiva del Desarrollo Humano promovida por él, como:

Una privación inaceptable de libertades sustantivas de los individuos, una condición de vida que limita sus capacidades para vivir la clase de vida que tienen razones para valorar. El individuo en situación de pobreza es aquel obligado a vivir una vida que no valora, obligado a sobrevivir, a subsistir. (p.1). 
En Venezuela, una de las características predominantes del entorno social, a lo largo del tiempo es la existencia de altos niveles de pobreza a pesar de las cuantiosas riquezas existentes. Moya (1975) señala que, desde el punto de vista económico, la Venezuela de 1830 hasta nuestros días se puede dividir en dos etapas: la Agropecuaria (1830-1926), eminentemente rural, con una producción agrícola orientada a la exportación; y la Venezuela Petrolera (1926Actualidad) con su acelerado proceso productivo rural-urbano, abandono del campo y con un incremento en los niveles de desigualdad y pobreza en el país. A su vez, vale la pena resaltar que la economía venezolana en las últimas tres décadas ha estado sometida a una inestabilidad recurrente. Los diferentes shocks, positivos y negativos, que la han impactado, y que la continúan impactando, y las respuestas de política económica (fiscal, monetaria, cambiaria, etc.) que los han acompañado, han afectado los niveles de ingreso y su distribución, y por esta vía, los niveles de pobreza. Estos shocks han sido básicamente petroleros, de precios de importaciones, de crisis de deuda externa y crisis financiera, con efectos directos sobre el nivel y estructura del producto interno, el nivel de empleo, la fuga de capitales, el ahorro y la inversión, el tipo de cambio real, los ingresos fiscales, la inflación y precios relativos, entre otros.

Entre uno de los objetivos fundamentales que se pretende alcanzar con el desarrollo pleno de esta investigación, es el de, proporcionar al lector un estado del arte conciso y completo, el cual engloba la revisión bibliográfica de algunos trabajos realizados en Venezuela y en otros países, acerca del fenómeno social objeto de estudio, bajo un análisis interdisciplinario y un enfoque multimétodo que permite abordarlo desde distintas concepciones científicas. Por otra parte, se abre el compás a una nueva discusión y discernimiento del fenómeno en el país, generando algunas ideas en paralelo con las presentadas por Harmath y Acevedo (2009), los cuales indagaron sobre el comportamiento de los niveles de pobreza total en Venezuela durante los años 1975-2000, haciendo uso de la técnica de regresión múltiple, considerando como variable respuesta la pobreza y como regresoras la tasa de inversión, el precio de realización del petróleo o precio de barril petrolero y el tipo de cambio promedio.

Este trabajo se encuentra estructurado en cinco secciones, incluyendo la presente introducción. La segunda, corresponde a la revisión bibliográfica, la cual tal y como fue mencionado anteriormente, engloba una serie de antecedentes relacionados con el problema de fondo lo cual permitirá establecer un horizonte de conocimiento a partir del cual se pueda discernir respecto a las diferentes metodologías y enfoques utilizados por otros estudiosos del tema objeto de estudio en Venezuela y otros países. En la tercera, se presentan algunos resultados empíricos obtenidos y una breve discusión de ellos, bajo el enfoque de regresión considerado; para así, tal y como fue mencionado en el parágrafo anterior, dar paso a una nueva concepción del problema de fondo, analizando el entorno bajo una visión diferente, buscando así, ampliar el horizonte de conocimiento en cuanto al tema objeto de análisis. Posteriormente, en la cuarta sección se presentan las conclusiones y recomendaciones que surgieron de esta investigación y se finaliza en la quinta parte con la bibliografía citada. 


\section{Revisión de literatura}

El objeto de estudio de este trabajo, ha sido protagonista en una gran cantidad de investigaciones. Esto ha permitido indagar sobre este flagelo, que se ha ido extendiendo arraigadamente en unos países más que en otros a lo largo del tiempo. El interés político y social que conlleva abordarlo, ha hecho que en diversos países del mundo incluyendo Venezuela, se le haya dedicado mucha atención al estudio de sus causas, determinantes y posibles políticas destinadas a su reducción.

Siguiendo las premisas (i), (ii), (iii) y (iv) referidas en la introducción; los integrantes del "Grupo Economía" del Decanato de Ciencias y Tecnología de la Universidad Centroccidental Lisandro Alvarado, partiendo del trabajo referido en la introducción, hemos seleccionado una serie de planteamientos de algunos autores e incluido otros, permitiendo analizar los diferentes enfoques utilizados para el estudio de la pobreza, para así, indagar acerca de sus posibles causas y algunas propuestas de sus potenciales soluciones.

Es necesario destacar que existen tres metodologías de medición de pobreza que son reconocidas a nivel mundial: i) Línea de Pobreza (LP): la más utilizada internacionalmente, toma el ingreso o gasto de consumo como medida del bienestar, establece un valor per cápita de una canasta mínima de consumo necesario para la sobrevivencia permitiendo así la diferenciación de los niveles de pobreza; ii) Necesidades Básicas Insatisfechas (NBI): toma en consideración un conjunto de indicadores relacionados con necesidades básicas estructurales (vivienda, educación, salud, infraestructura pública, etc.) que se requiere para evaluar el bienestar individual; iii) Medición Integrada: combina los métodos LP y NBI, clasifica a la población en cuatro grupos dependiendo de sus NBI y consumo: pobres crónicos, pobres recientes, pobres inerciales e integrados socialmente. En América Latina las metodologías más usadas son LP y NBI, sin embargo, organismos como el BID, la CEPAL y el BM establecen sus propias metodologías, basadas en la LP. (INEI, 2009).

Hopenhayn (1994), asegura que como fenómeno nada nuevo, la pobreza es una respuesta a efectos multicausales que se expresan en una diversidad de experiencias, cuyas rutinas más minúsculas reproducen los contrastes sociales y económicos en que viven los seres humanos. Para este autor, la presencia de la injusticia social y la dependencia, son las mayores y más dramáticas causas de la pobreza, al menos en los que respecta a Latinoamérica. A su vez, analiza las causas que tienen su origen en las oportunidades, y considera que las manifestaciones más relevantes se observan en la creciente brecha entre las expectativas de consumo y las cada vez menores posibilidades de colmarlas.

Por otra parte, expone que la visibilidad pública de la violencia como elemento cotidiano, instalado en los sectores más empobrecidos de las metrópolis latinoamericanas, incrementa el desprecio por la vida de seres con menos oportunidades de establecerse en condiciones de bienestar, seguridad e igualdad.

Igualmente, señala que en las sociedades latinoamericanas existe un contraste inmenso entre los beneficiados de los modelos de desarrollo (oportunidades, 
acceso y consumo) y los excluidos. Para estos últimos, la precariedad de su vida cotidiana es un drama que lo viven sin perspectivas de resolución porque, consideran que, no está en sus manos; la atribuyen, generalmente, al Estado o en su defecto a los gobiernos, trayendo como consecuencia el crecimiento entre populismo y pobreza. Todo esto, en Latinoamérica, ha generado una sensación de desesperanza que se ha instalado en los sectores más pobres, reflejándose ello en la proliferación de oficios de sobrevivencia y la aplicación de estrategias de "rebusque", utilizadas para no sucumbir a esas condiciones de vida.

López y Torres (1996), destacan que la concepción de la marginalidad vista como un polo excluyente, ha sido una base de suma importancia para los diversos enfoques sobre la problemática, en los cuales se analiza la diversidad de formas y recursos que las familias utilizan para enfrentar las condiciones de pobreza, especialmente en las zonas urbanas de los países latinoamericanos.

Para ellos, existen nuevas perspectivas en los estudios de pobreza que tienden a utilizar métodos económicos complementados con observaciones antropológicas y sociológicas, con la intención de incorporar las estructuras intermedias que se localizan en el nivel macro de la economía y en el nivel micro que se ha abordado por la antropología o la sociología utilizando metodologías cualitativas, dando cuenta de investigaciones ubicadas dentro de la perspectiva del análisis de la organización social, concebida como la síntesis entre lo individual y lo institucional.

Los autores plantean que los nuevos enfoques sobre la pobreza, perciben este fenómeno como un problema esencialmente moral y ético, siendo un asunto de derechos humanos 0 de ingobernabilidad potencial. Con respecto a los métodos de medición del flagelo, señalan que en los últimos tiempos los estudiosos de las ciencias sociales han realizado importantes esfuerzos para incorporar variables extra-económicas como las culturales, políticas, organizativas, psicológicas y religiosas, coincidiendo con la corriente del Pensamiento Complejo y considerando que el aporte de estas disciplinas, tienen un peso significativo en la comprensión de las múltiples dimensiones que constituyen este fenómeno, incidiendo ello profundamente en la modificación de estrategias empleadas para abordar la pobreza y superar los condicionantes que impulsan su reproducción.

Sen (1998), centra su trabajo en el desarrollo del bienestar social y la crítica a las interpretaciones de la teoría clásica de la economía del bienestar. Según él, esta teoría ha ignorado sistemáticamente el bienestar a largo plazo, o lo que se conoce como la tesis del desarrollo sostenible. Para este Nobel, la economía del bienestar y el sentido de cohesión social de grupos de identidades, están subordinados al desarrollo individual y al beneficio económico personal, siendo este último el objetivo de la racionalidad económica moderna. Tal planteamiento los hace sobre la discusión de las políticas económicas modernas del bienestar, las cuales según el autor, tienden a concentrar la atención en los ingresos, la riqueza y las utilidades.

Sen (ob. cit.) asume un enfoque diferente, el cual tiene que ver con la valoración de la pobreza, desde la perspectiva de evaluación de las desigualdades que emerge de esta consideración teórica del bienestar, sus efectos en la macroeconomía (efecto positivo) y en la microeconomía o 
bienestar de las personas (efecto negativo). Piensa que la evaluación de la desigualdad se encuentra íntimamente articulada con la valoración de la pobreza como la carencia de una realización mínima de algunas capacidades elementales, haciendo ello más fácil comprender por qué la pobreza tiene un aspecto absoluto y otro relativo. Esta afirmación viene dada en el sentido de que para él, es clave entender la naturaleza de la pobreza, pues no se concibe de igual manera la pobreza en un país rico como Estados Unidos que en un país pobre como Haití. Esta distinción que hace el autor, lleva implícita la importancia de la elección del espacio para identificar a los pobres y recolectar información sobre su situación y condiciones, pues asegura que "debe considerarse explícitamente la relación entre las privaciones en distintos espacios, especialmente entre espacio de los ingresos y el de la capacidad, cuando se quiere que todos gocen de vidas que valga la pena vivir". (p.21).

Por otra parte, O' Donnell (1999) sostiene que este fenómeno en Latinoamérica se remonta en la época colonial y a su vez, explica que la condición de pobreza está estrechamente vinculada a la escasez de bienes materiales y a la imposibilidad de superarla. Expone que, aún y cuando en algunos países de la región se observa un aumento en sus respectivos índices de crecimiento económico en la primera década del siglo XX, como es el caso de Brasil, Perú, México y Colombia, principalmente; tal crecimiento económico contrasta con los elevados índices de pobreza y desigualdad, que según Kliskberg (2008) suman más del $40 \%$, es decir, según el último citado casi la mitad de la población latinoamericana vive en la exclusión del bienestar material.

El autor desarrolla un enfoque de carácter histórico sobre la pobreza en América Latina, en cuyo anunciado advierte que esta es un legado colonial que dejó asentado en el continente un sistema social basado en la desigualdad étnica, siendo así un desequilibrio en las condiciones de vida de los descendientes europeos, los grupos indígenas sobrevivientes y los africanos traídos en condiciones de esclavitud. Para sustentar este argumento, acota que en países como México o Perú, los altos niveles de pobreza coinciden con las áreas pobladas por personas de origen indígena o africano, cuya exclusión se debe a las condiciones que han ocupado en la relación laboral establecida en el período colonial y que se ha reproducido hasta nuestros días.

Balza y Riutort (2001) estudian el comportamiento de los niveles de pobreza en Venezuela durante el período 1975-2000. Los autores hacen hincapié en la importancia que ha tenido el ingreso real como determinante de los niveles de pobreza del país, por ello en primera instancia, explican la influencia de algunos factores como la oferta de trabajo, el salario mínimo y otros determinantes sobre el salario real para posteriormente, analizar la influencia de ésta última variable sobre los niveles de pobreza considerándola como dependiente, todo ello a través de la estimación de algunos modelos econométricos de regresión múltiple.

Para uno de los modelos estimados, se obtiene que el aumento del $1 \%$ en el ingreso medio real de los hogares ocasionará una reducción del 1,02\% en los niveles de pobreza total de los hogares, bajo el supuesto que la distribución del ingreso no se altera y que a su vez, un aumento de $1 \%$ en el coeficiente de Gini producirá un aumento de $0,32 \%$ en el nivel de pobreza de los hogares, suponiendo que el ingreso real permanece constante. Otra de las estimaciones indica que un aumento de $1 \%$ en el salario real ocasionará una reducción de 
$0,91 \%$ en los niveles de pobreza total de los hogares del país ${ }^{5}$, bajo el supuesto que la distribución del ingreso no se altera. Por último, los autores concluyen, en base a los resultados obtenidos, que el salario real y la pobreza en Venezuela se relacionan en forma significativa con la composición del gasto público, los determinantes de la inversión privada, los términos de intercambio y el crecimiento de la fuerza de trabajo.

Riutort (2001) analiza la relación existente entre los niveles de pobreza, inflación y desempleo, determinando la magnitud de los efectos que sobre los niveles de pobreza han tenido, y pudieran tener; tanto el comportamiento de los precios como los cambios en la tasa de desempleo. A través de un proceso de simulación y sobre la base de la información suministrada por las Encuestas de Hogares por Muestreo del $\operatorname{INE}^{6}$ en Venezuela. El autor determina históricamente la influencia que tales variables ejercen sobre la magnitud de la pobreza durante el período 1982-1997 y su vez, predice el comportamiento probable de los niveles de la misma para los años 1998,1999 y 2000, bajo diferentes hipótesis de inflación y desempleo.

Según este autor, cada punto porcentual de desempleo tiene un mayor costo social en términos de pobreza que un punto porcentual de inflación, siendo este costo mucho mayor cuando se trata de los niveles de pobreza crítica. Por otra parte, concluye que bajo un escenario optimista del $13 \%$ de desempleo y $17 \%$ de inflación, la pobreza en el transcurso de los años 1999-2000 se habrá incrementado en por lo menos 7,9 puntos porcentuales (350.000 hogares) y la pobreza crítica en 4,7 puntos (207.000 hogares), impulsado el crecimiento de la pobreza en el año 2000, básicamente por una caída del salario real de aproximadamente 5\%; mientras que un escenario pesimista para la economía venezolana basada en la hipótesis de que el desempleo alcanza un porcentaje del 20\% y la inflación el 24\% pudiese ocasionar un aumento de hasta 14 puntos porcentuales (630.000 hogares) en los niveles de pobreza y un aumento cercano a los 10 puntos en los niveles de pobreza crítica (420.000 hogares).

Gontero et. al. (2001) indagan sobre algunas variables que inciden en la probabilidad de un hogar de ser o no pobre, mediante un análisis de regresión logística con el propósito de identificar perfiles de bienestar de la población de la Gran Córdoba de Argentina. Los autores, identifican los hogares pobres a partir del método de la línea de la pobreza absoluta utilizando los datos proporcionados por la Encuesta Permanente de Hogares realizada por el Instituto Nacional de Estadística y Censos (INDEC) en el mes de octubre del 2001.

Las estimaciones realizadas en la investigación citada, indican que las variables de mayor influencia en la probabilidad de ser pobre de los hogares cordobeses son el tamaño del hogar, la tasa de participación esperada, la edad del jefe del hogar, los años de educación promedio de las personas en edad de trabajar del hogar y los ingresos por hora por jefe del hogar, de ahí que concluyen que un hogar conformado por varios menores, con jefes de hogar

\footnotetext{
${ }^{5}$ En el estudio, la variable ingreso real está representada por el ingreso medio real de los hogares; mientras que el salario real corresponde al ingreso por persona ocupada, el cual se obtiene dividiendo el ingreso total de los hogares entre el total de personas ocupadas para un período determinado.

${ }^{6}$ Antes OCEI: Oficina Central de Estadística e Información.
} 
muy jóvenes y baja acumulación de capital humano constituye un grupo con alta probabilidad de pertenecer al conjunto de los hogares pobres.

Por último, recomiendan mejorar respecto a la asistencia en materia de salud reproductiva, lograr una mayor educación, conciencia y responsabilidad en la planificación del tamaño del hogar principalmente entre los jóvenes de grupos vulnerables. A su vez, confirman la importancia de la inversión en educación, advirtiendo la necesidad de implementar políticas educativas destinadas a lograr una mayor retención escolar y a mejorar la calidad de la enseñanza.

A su vez, Rodríguez (2002) sostiene que la mayoría de los países y organismos internacionales, destinan importantes recursos para generar investigaciones sobre este fenómeno que se traducen en formulación de políticas, aunque para él su efectividad sólo alcanza a la atenuación de esta anomalía social y no su erradicación.

En el análisis realizado por Rodríguez (ob. cit.) sobre los conceptos y enfoques de la pobreza, destaca que la gran mayoría de los trabajos tienen que sortear diferentes obstáculos vinculados a los marcos teóricos que asumen, o bien a los retos técnicos que representan los métodos empleados para caracterizar a los pobres.

Según este autor, generalmente las medidas que se adoptan para intervenir la pobreza no cubren las expectativas, atribuyéndole ello al hecho de que el empleo único de indicadores cuantificables quizás le resta importancia al empleo de indicadores del tipo cualitativo, siendo los últimos mencionados una valiosa herramienta que contribuye a la incorporación de factores que perfilan dimensiones cuya presencia es constante en los sistemas sociales en los cuales predomina la pobreza.

Boltvinick (2003) quien trabaja en la misma perspectiva de la pobreza de Amartya Sen, considera que este fenómeno tiene un origen multidimensional. Es decir, según este autor, la explicación de la pobreza está articulada con el empoderamiento del bienestar de los hogares y las familias, sugiriendo que para estudiar y comprender el flagelo propiamente dicho, es necesario hablar en plural de "las pobrezas", pues cualquier necesidad humana fundamental que no esté plenamente satisfecha revela una carencia, símbolo de pobreza.

En estos términos, Boltvinick (ob. cit.) asume como indicadores principales para estudiar la pobreza: el ingreso corriente, los activos no básicos, la capacidad de endeudamiento del hogar, los bienes y servicios durables que forman parte del patrimonio de la familia (vivienda, equipamiento del hogar), el acceso a bienes y servicios gratuitos (agua potable, entre otros), tiempo libre y el disponible para trabajos de tipo doméstico, educación y desarrollo del conocimiento de las personas, acceso a los satisfactores del entendimiento y desarrollo cognitivo del ser humano. En función del último argumento presentado, Boltvinick destaca entonces diferentes tipos del fenómeno entre los que se encuentran la pobreza de: (i) subsistencia, (ii) protección, (iii) afecto, (iv) entendimiento, (v) participación, (vi) ocio, (vii) creación, (viii) identidad y (ix) libertad.

Igualmente plantea que la fundamentación más adecuada para explicar las necesidades humanas debe buscarse en la antropología filosófica. Esto debido a que brinda un conjunto de herramientas para entender la naturaleza humana, y a entenderla es que se puede ser más asertivo en lo que se refiere a la 
identificación de cuáles son realmente las necesidades o carencias que hacen que un individuo, grupo o todo un sistema social viva o se considere en estado de pobreza.

Con estas afirmaciones, él, se acerca a una perspectiva multidimensional que permite abordar el estudio de las necesidades humanas. También, destaca que tales necesidades tienen un carácter histórico así como una dinámica cuya esencia e inclinación evolucionan hacia la universalización de los conceptos "pobreza y necesidades", los cuales, perfectamente pueden estar inmersos en un listado de bienes, servicios y actividades que son susceptibles de ser consideradas imprescindibles para preservar la vida y vivirla en condiciones que pueda generar satisfactores de esta necesidad en cualquier sociedad regida por la racionalidad científica moderna.

Por su parte, González (2005) analiza el comportamiento de los niveles de pobreza en Chile y Colombia, mediante la estimación de modelos de regresión lineal múltiple para así determinar en qué magnitud el incremento del flagelo ha estado influenciado por algunas variables demográficas, de política interna y de política externa como el tamaño poblacional, el gasto, la inflación y el grado de apertura económica durante el período 1970-2003.

Para el caso chileno, los resultados de la investigación infieren que las variables independientes explican el $79,65 \%$ de la variación de los niveles de pobreza e indican que los incrementos en la inflación y la apertura económica tienen un efecto directo sobre la variable endógena considerada, ya que un aumento de éstas implicaría un aumento en la pobreza de 0,0820 y 34,12 miles de personas respectivamente; mientras que un aumento del gasto pudiese disminuir la pobreza en 29,4132 miles de personas; no se infiere acerca de la influencia de la variable demográfica ya que no resulta estadísticamente significativa para el modelo estimado.

Respecto a Colombia, de manera similar fueron realizadas estimaciones empleando el método de mínimos cuadrados ordinarios; encontrando que para el modelo existe una alta relación explicativa entre la pobreza y las variables exógenas (explicativas) consideradas. En este caso, los resultados señalan que la inflación y la apertura económica tienen una relación positiva sobre el crecimiento de la pobreza; mientras que no se puede inferir acerca de la influencia del gasto gubernamental en la condición de pobreza de la población ya que la relación entre estas variables no resultaron significativas dentro del modelo estimado. Al analizar el comportamiento paralelo de ambos modelos de regresión estimados, para el caso de Chile se encuentra que, la apertura ha generado incrementos de pobreza, los cuales han sido anulados por aumentos en el gasto y disminución de la inflación; mientras que en Colombia aun y cuando la apertura también ha generado incrementos en la pobreza, la política interna establecida no ha logrado disminuir tal impacto.

Puente et. al. (2005) hallan ciertos determinantes de la pobreza, tanto general como extrema para Nicaragua, haciendo uso de la base de datos de la Encuesta de Hogares de ese país (EMNV 2001). La metodología empleada por los autores consiste en la estimación de un modelo de regresión logística y consideran como variable dependiente al nivel de consumo de un determinado hogar, transformándola en una variable del tipo binomial y usando el método 
de la línea de pobreza (general y extrema) estiman la probabilidad de que un hogar sea o no pobre.

Entre los principales hallazgos de Puente y otros (ob. cit.), se tiene que variables como hacinamiento, nivel de estudio, tipo de alumbrado, nivel de ingreso y total de miembros que consumen en el hogar, permiten estimar en forma apropiada la probabilidad de que un hogar se encuentre en condiciones de pobreza. A su vez, Puente y otros (ob. cit.) determinan que el área de residencia incide significativamente sobre la probabilidad de que un hogar sea pobre, siendo ésta más alta para aquellos hogares ubicados en áreas rurales y en los que hay condiciones de hacinamiento, e ubicados en zonas que no cuentan con alumbrado eléctrico y en donde el nivel de estudio del jefe es a lo sumo, primaria.

Phelan (2006), indica que las consecuencias de la complejidad de la pobreza, en las que se plantean diferentes ópticas y diversidad de conceptos es la creciente confusión entre las causas y los efectos que se originan en torno a la pobreza. Asegura que la multiplicidad de factores que originan este fenómeno, y su variabilidad según los grupos sociales en que se manifiestan, dificultan no sólo su comprensión sino también su explicación. Adicionalmente, afirma que para la comprensión de su significado se requiere identificar los factores que se destacan como causas y delimitarlos de los que se asocian a las consecuencias y así comprender cómo estos factores son articulados a través de relaciones dinámicas bajo diferentes circunstancias, generando de esta manera el mismo fenómeno.

Phelan (ob. cit.), señala que dentro de la diversidad de factores más señalados como generadores de pobreza destacan la falta de activos que limitan la adquisición de bienes y servicios adecuados para la reproducción económica (tierra, ganado, vivienda, fuerza de trabajo); y la falta de disponibilidad de capital financiero. Igualmente plantea que las sociedades deben preocuparse por el control de los activos sociales y políticos; así como también por alcanzar un elevado nivel de organización comunitaria, en vista de que la fortaleza de los activos ambientales y de infraestructura, inversión en el mantenimiento de las vías de comunicación, medios de transporte, agua, electricidad y demás activos, garantizan el bienestar de toda la población, de tal modo que en una sociedad en que la mayoría de las personas tienen un acceso limitado a estos recursos, bien sea porque son escasos o porque no tiene asignaciones presupuestarias suficientes, trae como consecuencia la falta de administración equitativa de la distribución de los bienes y de las oportunidades, agudizando la problemática.

Según estudios realizados por él en Venezuela ${ }^{7}$, los hogares ubicados en los estratos de la población más pobres, tienen un mayor número de personas dependientes (niños, adultos mayores, enfermos y discapacitados); en

\footnotetext{
${ }^{7}$ Para mayor profundidad en cuanto a investigaciones relacionadas con el tema de la pobreza en Venezuela, sugerimos al lector revisar con detenimiento los estudios desarrollados por el grupo Proyecto Pobreza de la Universidad Católica Andrés Bello (UCAB) presentados en Pobreza: Un mal Posible de Superar (2000) y Pobreza: Un Camino por Recorrer (2001), así como los libros Superar la Pobreza, Seguridad Social. Aportes para el Acuerdo (2002) y Detrás de la Pobreza. Percepciones. Creencias. Apreciaciones (2005), el primero de ellos elaborado en conjunto con algunos investigadores de la Unidad de Políticas Públicas (UPP) de la Universidad Simón Bolívar (USB).
} 
comparación con los hogares de los estratos de población menos pobres, intuitivamente concluyendo que a medida que la tasa de personas dependientes de las personas ocupadas del hogar sea menor, también será menor la probabilidad de que los hogares sean menos pobres. También argumenta que las regiones y municipios en Venezuela en donde hay mayores rasgos de fecundidad total y de iniciación reproductiva más temprana, menor prevalencia de uso de métodos anticonceptivos y mayor tendencia a la falta de planificación familiar y educación sexual no satisfecha, son justamente las zonas en las que se observan niveles de pobreza mucho más acentuados.

Por otro lado, Ugalde (2010) respecto a la pobreza en Venezuela expone que "la prosperidad de un pueblo no consiste en la cantidad de oro que posee, sino en el número de talentos y de brazos que emplea con utilidad" (p.10). Con esta afirmación Luis Ugalde, ex rector de la Universidad Católica Andrés Bello considera otra perspectiva de abordar este fenómeno en Venezuela, vinculada a la forma de gerenciar de los gobiernos latinoamericanos.

Este autor, destaca que la salida a la pobreza se avecinará una vez que se materialice una visión de país, la cual movilice todas las fuerzas del país logrando así una sólida educación, que contribuya a la transformación cultural productiva, en la que el país entero entienda que es a través del esfuerzo, del trabajo y de la responsabilidad social en conjunto, el camino que permitirá recrear condiciones y oportunidades novedosas, para convertir a los pobres en "productores y ciudadanos".

Para finalizar con el apartado, es claro que según los trabajos referidos, aún y cuando existen diferentes perspectivas del fenómeno las cuales coinciden en considerar la distribución desigual de los bienes materiales como el principal dispositivo de la pobreza; igualmente están presentes otras perspectivas las cuales, consideran que existen otros determinantes que no pueden ser dejados a un lado, a efectos de medir la posible presencia del flagelo en cualquier país incluyendo el nuestro.

\section{Posibles "lecturas" del flagelo}

El enfoque multimétodo, que según Gaviria, Mejía y Henao (2007) está "caracterizado por la complementariedad entre los datos cualitativos y cuantitativos, lo cual se logra utilizando el método de investigación-acción, llamado por algunos teóricos de triangulación o eclecticismo metodológico" (p.144) requiere el análisis separado de lo cuantitativo y lo cualitativo, para finalmente, comparar, evaluar y fusionar ambas tendencias y así concluir teniendo en cuenta esta metodología de investigación.

Brewer y Hunter (1990), citados por Gaviria, Mejía y Henao (ob. cit.), señalan que este enfoque se sustenta en el supuesto de que:

Las debilidades de cada método individual han de ser compensadas por la fortaleza de otro método. Se asume que los métodos tienen imperfecciones o debilidades distintas, y que el abordaje multimétodos usa como estrategia tratar 
el problema a investigar con un arsenal de métodos sin superponer debilidades, agregando las ventajas complementarias. (p.145)

Es por ello que esta sección, se presenta dividida en los enfoques cuantitativo y cualitativo, para así, proporcionar al lector la triangulación de las ideas generadas arriba, siendo ellas condensadas y ampliadas en el apartado de implicaciones de políticas económicas y arquetipo venezolano.

\subsection{Enfoque cuantitativo}

Debido a la naturaleza de las variables incluidas en la investigación de Acevedo y Harmath (ob. cit.), previamente a la etapa de estimación del modelo econométrico, fueron considerados una serie de pasos con el objetivo de afianzar los resultados y evitar regresiones espurias; entre los que destacan las pruebas de raíz unitaria y las pruebas de cointegración de las variables (series temporales) objeto de estudio ${ }^{8}$.

Se determinó que las variables pobreza total ${ }^{9}$ bajo transformación logarítmica ${ }^{10}$, la tasa de inversión, el precio de realización del petróleo y el tipo de cambio promedio alcanzan un nivel estacionario en media y varianza, al ser tomadas en primera diferencia, bajo la influencia de algunos factores exógenos como patrones determinísticos o de tendencia. Por otra parte, se encontró que tales series temporales cointegran, y por consiguiente mantienen una relación de equilibrio en el largo plazo, en el sentido de que hay al menos una combinación lineal de las series involucradas en el sistema la cual estacionaria, por supuesto, para el período objeto de análisis.

A partir del modelo econométrico propuesto y estimado, se obtuvieron una serie de resultados relevantes con la finalidad de contrastar en ese entonces, un conjunto de hipótesis establecidas en la investigación. En la expresión que sigue:

$$
L P O B_{t}=\beta_{0}+\beta_{1} T I_{t}+\beta_{2} P R P_{t}+\beta_{3} T C_{t}+\varepsilon_{t}
$$

$L P O B_{t}$ corresponde a los niveles de pobreza total bajo transformación logarítmica (natural) para el período considerado, $T I_{t}$ la tasa de inversión, $P R P_{t}$

\footnotetext{
${ }^{8}$ Ver Anexos 1, 2 y 3.

${ }^{9}$ Los datos utilizados en este estudio respecto a los niveles de pobreza total para el período considerado, se refieren a estimaciones realizadas por Balza y Riutort (2001) a partir de los datos provenientes de la Encuesta de Hogares por Muestreo (EHM) realizada semestralmente por el Instituto Nacional de Estadística (INE). Los mismos emplearon el Método de Línea de Pobreza (LP) considerando el hogar como unidad de muestreo, para realizar las debidas estimaciones (obtenidas en porcentajes) considerando cada uno de los hogares empadronados en el primer semestre de cada año, una vez aplicada la encuesta.

${ }^{10}$ Antes de ser aplicado el logaritmo neperiano, la serie original expresada en porcentaje fue transformada, llevándola a totales de personas en situación de pobreza total para cada año en particular, usando los datos de población total venezolana disponibles en el portal electrónico Penn World Table 6.2, realizando simplemente una transformación lineal de la serie original, todo ello por razones de interpretación de los parámetros a estimar en función de la variable dependiente. Expresar ésta última como la tasa (logaritmo) de un porcentaje, en cierta forma carece de sentido desde el punto de vista económico.
} 
el precio del barril petrolero o el precio de realización del crudo , $T C_{t}$ el tipo de cambio promedio, $\beta_{0}$ representa el término constante o el intercepto del modelo y $\varepsilon_{t}$ el de perturbación aleatoria. Es importante dejar claro que se consideraron varios modelos tentativos, uno de ellos incluyendo como variable independiente la pobreza rezagada un período; mientras que para otro se incluyó ésta rezagada dos períodos; sin embargo, gran parte de los parámetros estimados no presentaron el comportamiento esperado desde el punto de vista estadístico y económico ${ }^{11}$. Los resultados obtenidos para los modelos referidos anteriormente, así como los del modelo ${ }^{12}$ presentado en (1) se muestran a continuación:

Cuadro 1. Resultados de la Estimación

\begin{tabular}{|c|c|c|c|}
\hline \multicolumn{4}{|c|}{ Variable Dependiente: LPOB } \\
\hline $\begin{array}{c}\text { Variable } \\
\text { Independiente }\end{array}$ & (1) & (2) & (3) \\
\hline C & $\begin{array}{l}17,2749 \text { *ᄎ* } \\
(72,3465)\end{array}$ & $\begin{array}{c}1,3861 \\
(1,0666)\end{array}$ & $\begin{array}{c}1,5127 \\
(0,6157)\end{array}$ \\
\hline TI & $\begin{array}{l}-0,0505^{\star \star \star} \\
(-7,9814)\end{array}$ & $\begin{array}{l}-0,0070 * \\
(-1,7628)\end{array}$ & $\begin{array}{l}-0.0101 \\
(-1,5067)\end{array}$ \\
\hline PRP & $\begin{array}{l}-0,0243^{\star * *} \\
(-2,9100)\end{array}$ & $\begin{array}{c}0.0046 \\
(1,2033)\end{array}$ & $\begin{array}{c}0.0085 \\
(1,1296)\end{array}$ \\
\hline TC & $\begin{array}{l}0,0006^{\star *} \\
(2,5541)\end{array}$ & $\begin{array}{c}-0.0001 \\
(-1,3202)\end{array}$ & $\begin{array}{c}-0.0003 \\
(-1,5155)\end{array}$ \\
\hline LPOB(-1) & - & $\begin{array}{l}0,921^{\star \star \star} \\
(12,2732)\end{array}$ & - \\
\hline LPOB(-2) & - & - & $\begin{array}{c}0.9169 \\
(6,4406)\end{array}$ \\
\hline
\end{tabular}

${ }^{11}$ La información estadística respecto a las variables tasa de inversión (\%), precio de realización del petróleo (US\$/b) y tipo de cambio promedio Bs/US\$) tiene como fuente el Informe Académico y Anuario de Cuentas Nacionales, publicado por el Banco Central de Venezuela (BCV) en el año 2001.

${ }^{12}$ Para una mayor comprensión de las bases teóricas del mismo, véase el Apéndice A. 


\begin{tabular}{|c|c|c|c|}
$\mathbf{R}^{2}$ & 0,8429 & 0.9824 & 0.9506 \\
$\mathbf{R}^{2}$ ajust. & 0,8215 & 0.9788 & 0.9402 \\
$\mathbf{F}$ & 39,3727 & 279.1140 & 91.5492 \\
$\mathbf{D W}$ & 1,5473 & 1.6357 & 1.2590 \\
$\begin{array}{c}\text { Número de } \\
\text { observaciones }\end{array}$ & 26 & 25 & 24 \\
\hline
\end{tabular}

$\mathbf{R}^{2}$ : Coeficiente de Determinación. $\mathbf{R}^{2}$ ajust. : Coeficiente de Determinación ajustado. F: Estadístico F de Snedecor. DW: Estadístico Durwin-Watson. Hipótesis Nula "El parámetro estimado es significativo". Regla de decisión: si $p>\alpha$ no se rechaza la Hipótesis Nula. Si $p<\alpha$ se rechaza. $\alpha=0,1 ; 0,05$ y 0,01 para los niveles $10 \% ; 5 \%$ y $1 \%$ respectivamente. *, ${ }^{* *} \mathrm{y}$ *** denotan rechazo de la Hipótesis Nula al $10 \%$, $5 \%$ y $1 \%$. Estadísticos t en ()

Fuente: Harmath y Acevedo (2009)

La estimación del modelo original planteado en la ecuación (1) indica que la tasa de inversión, los precios de realización del petróleo y el tipo de cambio promedio explican el $84,29 \%$ de la variabilidad observada para los niveles de pobreza total observados para Venezuela en el período temporal en estudio $\left(R^{2}=0,8429\right)$. Adicionalmente se tiene que el valor del estadístico $\mathrm{F}(39,3727)$ permite indicar que las variables en su conjunto son adecuadas para explicar la dependiente y los parámetros estimados para las variables explicativas antes mencionadas son estadísticamente significativos. A su vez, el estadístico Durwin Watson se aproxima a $2(1,5473)$ lo cual es una evidencia suficiente para determinar que no hay patrones de correlación serial para los residuos de la regresión, problema el cual se presenta con bastante frecuencia cuando se estiman modelos de este tipo utilizando datos de corte temporal.

Por otra parte, se tiene los signos observados para los parámetros estimados son los esperados, y por ello, el comportamiento entre la variable dependiente respecto a cada de las independientes del sistema es totalmente congruente con la teoría económica. De este modo, los coeficientes indican que un aumento del 1\% en la tasa de inversión ocasionaría una reducción del 5,05\% de la tasa de crecimiento de la pobreza total de los venezolanos, bajo el supuesto que el precio de realización del petróleo y el comportamiento del tipo de cambio promedio en el mercado no se alteren. A su vez, un aumento de $1 \$$ en los precios del barril de petróleo en el mercado produciría un decremento o reducción de la tasa de crecimiento de la pobreza del 2,43\%, bajo el supuesto 
que la tasa de inversión y el tipo de cambio promedio permaneciesen constantes (ceteris paribus); mientras que un incremento de Bs. 1 en el precio del $\$$ produciría un aumento en la tasa de crecimiento de la pobreza total venezolana del $0,06 \%$ siempre y cuando la tasa de inversión y el precio de realización del petróleo permaneciesen constantes en el mercado.

\subsection{Enfoque cualitativo}

Para indagar acerca de los niveles de pobreza total en Venezuela, desde una perspectiva cualitativa, se debe tener en cuenta que hay otros factores presentes en la sociedad venezolana, que pueden convertirse en elementos claves para la comprensión y el análisis del flagelo antes mencionado en nuestro país.

Se debe hacer énfasis en determinadas variables cuyo comportamiento le asignan particularidades que pueden considerarse influyentes respecto a la permanencia de la pobreza en el proceso social venezolano. Es a partir de esta reflexión, que surge la necesidad de incorporar otros puntos de vista que contribuyan a su abordaje, haciendo uso de herramientas teóricometodológicas enmarcadas dentro de la corriente del Pensamiento Complejo, cuya concepción pueda dar cuenta de las múltiples dimensiones, mediante las cuales se pueda identificar y explicar la pobreza en el contexto de la Venezuela contemporánea.

En concordancia con algunos teóricos presentados en la sección 2, hubo un cambio de paradigma influyente en la evolución de la sociedad venezolana desde el inicio del período de la "cultura petrolera", afianzada en el país con los modelos de desarrollo adoptados a partir de la segunda guerra mundial. Momento histórico en el cual, todas las sociedades latinoamericanas, la venezolana particularmente, fueron impactadas por la noción de desarrollo vinculada al progreso y bienestar económico de la sociedad en su conjunto.

Durante la década de los años 20 , una buena parte de los venezolanos se encontraban inmersos en la más absoluta pobreza. Esto, como consecuencia de la precariedad que revestía la actividad agropecuaria, principal fuente de sustento para la mayoría de la población. La falta de asistencia y la ausencia de un plan económico nacional, colocaba al país entre los más pobres del continente.

En ese período, el ingreso per cápita en Venezuela se estimaba 30\% menos que en los demás países latinoamericanos. Existía una sociedad predominantemente rural, la mayoría de sus habitantes tenía una muy baja calidad de vida, el país presentaba un elevado índice de analfabetismo y poseía escasas posibilidades de capacitación y grandes rasgos de insalubridad. Estas características fueron las razones para querer incorporarse a los procesos de industrialización que exigían los modelos de desarrollo.

La aparición del petróleo cambia progresivamente la estructura de la sociedad venezolana. La nueva realidad económica exigía un desplazamiento de la masa trabajadora hacia las zonas donde se requería mano de obra que se pudiese ocupar en el incipiente proceso de industrialización; no sólo en las áreas directamente vinculadas a la explotación petrolera. Según Baptista 
(1984) este es un fenómeno que durante largas décadas, se mantuvo en a la vida nacional.

El fenómeno de la migración rural-urbana, se produjo hacia las zonas que potencialmente eran aptas para desarrollar un amplio mercado tanto al interior del país, como para conectarse con el mercado internacional; explicando ello la elevada concentración poblacional de las regiones Capital, Central y CentroOccidental (las cuales concentran las ciudades Distrito Capital, Maracaibo, Valencia, Barquisimeto, Maracay, Miranda, entre otras).

Ello, por un lado, produjo un desequilibrio en la densidad poblacional de los estados que conformaban el territorio venezolano. Por otra parte, el acelerado proceso productivo no canalizado correctamente desde el punto de vista económico-social por los gobiernos de turno, generó un "sentimiento de vulnerabilidad" en gran parte de los habitantes urbanos migrantes de las zonas rurales durante el período del boom petrolero. Al verse "excluidos progresivamente" de los beneficios económicos y sociales generados por la renta petrolera, se desató una creciente insatisfacción social, económica, política y cultural, siendo esto una característica común en las economías donde falla la distribución racional y equitativa de los ingresos y riqueza de la nación. Todo esto propició un escenario cuasi-perfecto para justificar o motivar los altos niveles de desigualdad, pobreza e inseguridad que se han mantenido en constante crecimiento hasta la actualidad.

\subsection{Implicaciones de políticas económicas y arquetipo venezolano}

Por lo expresado arriba, el análisis ahora presentado en este trabajo, a diferencia del que se realiza en investigaciones exclusivamente cuantitativas o cualitativas, cubre la llamada triangulación multimétodo. De esta manera, se abordó el flagelo objeto de análisis de una manera más compleja e integral que desde un solo enfoque.

Teniendo en cuenta lo planteado anteriormente; en primer lugar, se puede inferir la importancia que tienen las variables independientes analizadas por Harmath y Acevedo al momento de establecer políticas económicas que vayan dirigidas a la disminución de la pobreza. En tal contexto, los gobiernos deberían incentivar la inversión puesto que es uno de los determinantes que más impacta en la reducción de la tasa de crecimiento de la pobreza total. De esta manera se lograría reactivar la economía; y las consecuencias de una mala distribución de las riquezas del país comenzarían a subsanarse por medio del incentivo del sector privado. Igualmente, esto permitiría diversificar los sectores económicos del país, lo cual conllevaría a mejorar las condiciones de estado mono-productor y la densidad poblacional pudiera comenzar a equilibrarse y repartirse proporcionalmente no sólo en las regiones petroleras.

A su vez, los encargados de administrar la producción petrolera deberían establecer políticas que permitiesen mantener un precio del barril estable o preferiblemente al alza, y los generadores de políticas cambiarias velar por mantener el tipo de cambio estable. Sin embargo, a pesar que las estimaciones analizadas sustentan la influencia del precio de realización del petróleo sobre la tasa de crecimiento de pobreza, las erradas políticas de distribución de los ingresos petroleros han contribuido notablemente al hecho que más de la mitad 
de los habitantes del país quedaran prácticamente rezagados de los beneficios de la renta generada por concepto de las exportaciones, encontrándose que el $55,1 \%$ de las personas se encontraban en situación de pobreza ${ }^{13}$ durante el período 1970 - 1981, cuando el precio del petróleo aumentó de 1,84US\$ a 29,71US\$.

Adicionalmente, vale destacar que al momento de implementar políticas dirigidas a la disminución de la pobreza, el gobierno debería apuntar hacia la inversión incentivando a la privada y aumentando la pública, debido a que éste es el determinante sobre el que mayor influencia tiene, ya que, el precio del barril del petróleo y el tipo de cambio a pesar de poder administrarse con cautela para que no sufran un shock negativo, responden más a variables exógenas como la oferta y la demanda en el mercado internacional y/o nacional. Al igual se debería subsanar las fallas de la distribución de la riqueza proveniente del petróleo.

Ahora bien, debido a la complejidad del fenómeno objeto de análisis, más allá de la influencia bien sea positiva o negativa que ejercen o pueden ejercer sobre él, determinadas variables macroeconómicas; es innegable que la visión de Ugalde es compartida con muchos críticos de la "cultura petrolera" mencionada anteriormente, incluyéndonos. Es evidente pensar que el cambio de paradigma al cual nos referimos en 3.2, ha trastocado notablemente los valores y la visión de sí mismo de los venezolanos.

La sociedad venezolana debería ser capaz de entender que, es la concepción de un sólido y profundo sistema "moral-educativo" uno de los dispositivos de lucha más sanos contra los preocupantes niveles de desigualdad y pobreza observados en nuestro país; ya que por un lado, es natural esperar que a medida que aumenten los niveles de escolaridad de un hogar determinado, aumente la probabilidad de que tal hogar no se considere pobre, en particular porque a medida que aumente la escolaridad debería aumentar la capacidad de generar mayores ingresos. Además, quizás se logre concientizar profundamente a un grupo significativo de la población, especialmente a aquellos comprendidos en los estratos sociales más bajos, en términos de un control adecuado de natalidad, a efectos de disminuir la peligrosa cifra de embarazos precoces observada en Venezuela año tras año.

Igualmente, consolidar un sistema de salud plausible es fundamental, ya que la salud es un factor determinante para disminuir la pobreza. En este contexto, aquellas sociedades que han alcanzado un nivel de vida equilibrado, consideran la salud como el principal bien que el Estado debe garantizar a toda la población, pues si esta necesidad no es satisfecha es muy probable que sea una sociedad con grandes sociedades para mantener elevada su productividad, implicando así que haya un crecimiento de los grupos más vulnerables con respecto a las condiciones de pobreza.

Ahora, si bien el Estado debe ser un poco más profundo en cuanto a las estrategias sociales aplicadas en materia de educación, empleo, salud, inseguridad; entre otras, debería ser más rígido en cuanto al cumplimiento de determinadas normas por parte de sus ciudadanos, apuntalando siempre a alcanzar sus metas de integración y desarrollo de intereses comunes.

\footnotetext{
${ }^{13}$ Según cifras del Ministerio de Planificación y Desarrollo.
} 
Generalmente el no cumplimiento de normas se presenta con mayor frecuencia en aquellos estratos sociales con menor desarrollo de oportunidades y con fuertes obstáculos, los cuales obviamente dificultan el desarrollo pleno de sus capacidades; y lastimosamente, Venezuela no escapa de tal realidad.

\section{Conclusiones y recomendaciones}

El presente artículo, emergió para satisfacer la necesidad de un análisis exhaustivo del estado del arte de un tema tan abordado como la pobreza. Se elaboró bajo un enfoque multimétodo, el cual a pesar de ser duramente criticado por algunos teóricos netamente cualitativos 0 cuantitativos ${ }^{14}$, pensamos, es la mejor alternativa para esta investigación. Esto se debe, a que permite un abordaje más amplio e integral que se sustente en los análisis cuantitativos y cualitativos realizados. De esta manera, se puede tener un corpus teórico-metodológico que permitirá lograr los objetivos del proyecto de investigación del cual forma parte. Así, el uso de herramientas enmarcadas dentro de la teoría econométrica clásica y la corriente del Pensamiento Complejo en este artículo, permitieron identificar y explicar la pobreza en el contexto de la Venezuela contemporánea desde múltiples dimensiones; entre ellas: i) económica, ii) desarrollo humano, iii) exclusión socio-políticoeconómica, iv) valores humanos. Esto permitió, generar en paralelo, ampliar, respaldar, sustentar desde el enfoque cualitativo y profundizar algunas ideas con las presentadas por Harmath y Acevedo (2009).

En cuanto a las implicaciones de políticas económicas, queda claro que no sólo basta con la implementación de programas o estrategias direccionadas al entorno económico. La dimensión de Desarrollo Humano, es una de las más resaltantes así como la de exclusión y valores humanos. Esto, nos lleva a recomendarle a los gobiernos de turno, tener en cuenta que las políticas económicas destinadas a la disminución de la pobreza, no tendrán un impacto de largo plazo si sólo cubren la necesidad económica del corto plazo. En palabras de Lao Tse "Si estimas a alguien, y ves que tiene hambre, no le des un pescado, enséñale a pescar. Porque si le das un pescado lo alimentarás un día, pero si le enseñas a pescar lo alimentarás para toda la vida", este proverbio muy trillado, hasta en distintas religiones, es ideal para la realidad actual venezolana.

Los gobiernos de turno no deberían pretender suplir todas las necesidades económicas en el largo plazo. Esto, debido a que induce significativamente a la gestación de ciudadanos pasivos en cuanto a la fuerza laboral y productiva. Esquemas económicos-socio-capitalistas como el de Noruega, son sistemas dignos de replicar en este país que posee una riqueza petrolera y natural bastante privilegiada. Sin embargo, el implementar este tipo de sistema

\footnotetext{
${ }^{14}$ Por razones obvias, los teóricos que sienten esta posición no son citados. Se consideró irrelevante, ya que este artículo versa sobre la pobreza y no sobre la teoría de la metodología utilizada, sin embargo sí se consideró necesario incluir este comentario para justificar su uso. Si se quiere profundizar sobre la teoría metodológica de este enfoque, se recomienda, entre otros, Gaviria, Mejía y Henao (2007).
} 
conlleva a un cambio radical de paradigmas socio-políticos, económicos y hasta culturales que sólo se pueden lograr por medio de un sistema educativo de formación de valores e identificación nacional. Con lo antes planteado, no se quiere decir que el gobierno nacional debe abstenerse de velar por el bienestar económico de sus ciudadanos. Políticas que subsidien "temporalmente" en el corto plazo, la cesación laboral por medio de un sistema que permita la distribución equitativa, justa y racional de la riqueza nacional, mientras otras políticas reactivan el aparato productivo (público y privado) el cual, debería absorber esa "carga económica improductiva" en un período no mayor a un mediano plazo, son factibles y muy necesarias. El meollo del asunto radica, en el hecho que no se puede justificar, bajo ningún pretexto; ni alegando la igualdad de condiciones o género que un "hogar improductivo ${ }^{15}$ " genere unos ingresos iguales o hasta superiores que un "hogar productivo ${ }^{16 "}$. Esto, debido a que en vez de incentivar al desarrollo humano y a la siembra de valores, se tergiversa en la condición del hombre y mujer como un animal racional, político, económico que busca su beneficio y confort propio al menor costo de oportunidad. Si bien, las políticas de ayudas sociales han logrado en cierto modo, disminuir ese "sentimiento de vulnerabilidad" en los sectores más desfavorecidos de la población, es probable que ese sentimiento haya aumentado en sectores que anteriormente no lo tenían.

Finalmente, es fundamental acotar que, más allá de hacer referencia a algunas implicaciones de políticas económicas, consideradas a partir de las estimaciones obtenidas; es importante destacar que el abordaje realizado de la pobreza total en Venezuela, desde la perspectiva tradicional nos ubica en el proceso de complejización del fenómeno en estudio, así como en la identificación de particularidades que le agregan al análisis y elementos que pueden vislumbrarse como estimaciones que le asignan un carácter profundamente social y humano a uno de los problemas más difíciles de abordar; y por ende, no resulta nada fácil alcanzar respuestas consistentes que nos ayuden a incorporar alternativas válidas para superar el flagelo que nos agobia y que cada vez se complejiza más su presencia en nuestra sociedad. Debe tenerse presente que existen múltiples factores que, de acuerdo al contexto en que surgen, adquieren características que no son mensurables empleando algunos métodos clásicos, y es por esta razón que en la continuidad del estudio sobre la pobreza total en Venezuela, incorporamos otros aspectos cualitativos, los cuales consideramos que contribuyen a profundizar acerca de la presencia de este fenómeno en la realidad venezolana. De ahí que más adelante, es obvio que en un corto plazo, el equipo de trabajo se focalizará en incorporar indicadores que den cuenta de las múltiples dimensiones desde las cuales se pueda identificar y explicar los determinantes de la pobreza en el país; por supuesto, pasando por abordar el tema objeto de estudio desde el punto de vista cuantitativo, con datos más recientes y bajo diversas metodologías, claro está, agarrado ello de la mano con una serie de aspectos cualitativos los cuales no pueden ser dejados a un

\footnotetext{
${ }^{15}$ Entiéndase un hogar conformado por personas que no laboran (madres solteras, presos, becarios de las misiones sociales, entre otros), pero que por subsidios gubernamentales obtienen un ingreso mensual.

${ }^{16}$ Entiéndase un hogar donde al menos uno de sus integrantes trabaje formalmente en la economía y su salario sea el sustento del mismo.
} 
lado, al momento de indagar acerca de este fenómeno tan complejo y arraigado a nivel mundial.

\section{Referencias}

Acevedo, R. y Harmath, P. (2009). "Determinantes económicos de la pobreza total en Venezuela: 1975-2000". Economía, No 28, 2, pp. 161-189. Revista del Instituto de Investigaciones Económicas y Sociales. Facultad de Ciencias Económicas y Sociales. Universidad de Los Andes. Mérida, Venezuela.

Balza, R. y Riutort, M. (2001). "Salario Real, Tipo de Cambio Real Pobreza en Venezuela". Temas de Coyuntura, 44, Caracas: Universidad Católica Andrés Bello, pp. 5-68.

Baptista, A. (1984). "Más allá del Optimismo y del Pesimismo: las Transformaciones Fundamentales del País". En el caso Venezuela. Una ilusión de armonía. Ediciones IESA. Caracas Venezuela, pp. 20-41.

Boltvinick, J. (2003). "Tipología de los Métodos de Medición de la Pobreza. Los Métodos Combinados". Revista Comercio Exterior: Vol. 53, № 5 . Banco de Comercio Exterior. México. D.F.

Gaviria, M., Henao, D. y Mejía, A. (2007). "Gestión de Investigación en los grupos de Investigación de excelencia de la Universidad de Antioquia". Revista Interamericana. Biblioteca de Medellín. Colombia. Vol. 30 N² 2, pp. 137 - 163.

Gontero, S., Ojeda, S. y Pereyra, L. (2005). "La pobreza en los hogares del modelo del Gran Córdoba: aplicación del modelo de regresión logística". Revista de Economía y Estadística: Vol. XLIII, 1. Argentina: Instituto de Economía y Finanzas, Facultad de Ciencias Económicas, Universidad Nacional de Córdoba.

González, P. (2005). “Integración Económica y Pobreza Nacional: El caso de

Colombia Colombia, Chile y México (1980-2002)". Ponencia presentada en el XX Congreso Nacional de estudiantes de economía, globalización y desarrollo de la Federación Nacional de Estudiantes de Economía. Colombia.

Heston, A., Summers, R. y Aten, B. (2006). Penn World Table Version 6.2. Center for International Comparisons of Production, Income and Prices at the University of Pennsylvania.

Hopenhayn, M. (1994). Ni Apocalípticos Ni Integrados. Editorial Fondo de Cultura Económica. Santiago de Chile.

Instituto Nacional de Estadística e Informática (2009). Documento en línea. Disponible en: http://www1.inei.gob.pe/biblioineipub/bancopub/Est/Lib0329/indiceTM. Consultado el 02 de noviembre de 2009.

Ministerio de Planificación y Desarrollo: Cumpliendo las Metas del Milenio, Anexo Pobreza, p. 89 
Molina, E. (2009). La identificación y medición de la Pobreza desde la perspectiva del Desarrollo Humano. Documento en línea. Disponible en: http://www.flacso.or.cr/fileadmin/user_upload/biblioteca_digital/CURSO_VIRTU AL/CPmedicion_pobreza.pdf. Consultado el 30 de octubre de 2009.

López, R. y Torres, G. (1996). La pobreza rural: hacia un enfoque multidimensional. Disponible en: Carton de Grammont, H. Nuevos Procesos rurales en México.

O Donell, G. (1999). Pobreza y Desigualdad en América Latina. Disponible en: Pobreza y Desigualdad en América Latina. Temas y Nuevos Desafíos. Editorial Paidós. Argentina.

Phelan, M. (2007). La Desigualdad en Venezuela. Fundación Escuela de Gerencia Social. Caracas. Documento en línea. Disponible en: http://fegs.msinfo.info/opac/php/inicio. Consultado el 15 de noviembre de 2011.

Puente, L., Sananes, M., Sinha, S. y Torres, E. (2005). "Determinantes de Pobreza en la Encuesta de Hogares, Nicaragua 2001 Usando Regresión Logística". Póster presentado en el II Encuentro Binacional de Estadística realizado en la Facultad de Ciencias Económicas y Sociales de la Universidad de Los Andes, Mérida, Venezuela.

Riutort, M. (2000). Inflación, Desempleo y Pobreza en Venezuela. Borradores de

Trabajo: La Pobreza en Venezuela. Causas y Posibles Soluciones $\mathrm{N}^{\circ} 14$. Caracas: Universidad Católica Andrés Bello, Instituto de Investigaciones Económicas y Sociales.

Rodríguez, H. (2002). Enfoques para la Medición de la Pobreza. Documento en Línea. Disponible en: www.mty.itesm.mx/egap/centros/caep/2_2htm. Consultado el 15 de noviembre de 2011.

Sen, A. (1998). Nuevo Examen de la Desigualdad. Alianza Editorial. Ciencias Sociales. España.

Ugalde, L. (2010). Salir de la Pobreza. Artículo de opinión publicado en El Nacional, domingo, 31 de octubre de 2010. Caracas, Venezuela. 
6. Anexos

Anexo 1. Test de Raíz Unitaria

Series en niveles

\begin{tabular}{|l|ccc|c|c|c|}
\hline & \multicolumn{3}{|c|}{ ADF } & \multicolumn{3}{c|}{ PP } \\
\hline & I & II & III & I & II & III \\
\hline POB & -2.0105 & -0.7036 & 0.8735 & $-1,1430$ & $-1,1721$ & 0,7638 \\
TC & 4.7694 & 4.5223 & 4.7535 & 2,7316 & 0,5309 & 3,7132 \\
PRP & $-2,0360$ & -2.0176 & -0.0012 & $-2,0325$ & $-1,9401$ & 0,0758 \\
TI & $-3,0642^{\star *}$ & $-2,5532$ & $-2,3547^{\star *}$ & $-1,4042$ & $-2,5377$ & $-1,5842$ \\
LPOB & $-1,2815$ & $-0,1993$ & 0,7675 & $-1,1925$ & $-0,9758$ & 1,3524 \\
LTC & 1,4621 & $-2,3779$ & 4,7378 & 1,0576 & $-2,4642$ & 3,7355 \\
LPRP & $-2,2747$ & $-2,2527$ & 0,4003 & $-2,1928$ & $-2,0940$ & 0,5269 \\
LTI & $-1,9729$ & $-3,2061$ & $-1,8556^{*}$ & $-1,6889$ & $-3,0989$ & $-1,3752$ \\
\hline
\end{tabular}

ADF: Augmented Dickey Fuller; PP: Phillips Perron.

Los rezagos para el ADF fueron predeterminados por el E-Views 6.0 (Max. 5) bajo Criterio Akaike.

I: Intercepto; II: Intercepto y Tendencia; III: Sin variable exógena.

Hipótesis Nula para ADF: "La serie tiene raíz unitaria"

Regla de decisión: si $p>\alpha$ no se rechaza la Hipótesis Nula. Si $p<\alpha$ se rechaza. $\alpha=0,1 ; 0,05$ y 0,01 para los niveles $10 \%, 5 \%$ y $1 \%$ respectivamente. *, ** y *** denotan rechazo de la Hipótesis Nula al $10 \%$, $5 \%$ y $1 \%$

Series en primeras diferencias

\begin{tabular}{|c|c|c|c|c|c|c|}
\hline & \multicolumn{3}{|c|}{ ADF } & \multicolumn{3}{|c|}{ PP } \\
\hline & $I$ & II & III & $I$ & II & III \\
\hline POB & $\begin{array}{c}- \\
4,1551^{\star \star \star}\end{array}$ & $-1,4419$ & $\begin{array}{c}- \\
3,8464^{\star \star *}\end{array}$ & $-2,9556^{\star}$ & $-2,8670$ & $-2,9643^{\star \star \star}$ \\
\hline TC & $-3,1100^{\star \star}$ & $\begin{array}{c}- \\
4,4928 \star \star \star\end{array}$ & $-1,2372$ & $-3,3295^{\star \star}$ & $-4,4804^{\star \star \star}$ & $-2,7643^{\star \star \star}$ \\
\hline PRP & $\begin{array}{c}- \\
3,8671^{\star \star \star}\end{array}$ & $-3,7242^{\star \star}$ & $\overline{-}^{-}$ & $-3,7149 * *$ & $-3,5517^{*}$ & $-3,7452^{\star * *}$ \\
\hline TI & $\begin{array}{c}- \\
5,3855^{\star \star \star}\end{array}$ & ${ }^{-}$ & ${ }^{-}$ & $-6,0285^{\star \star \star}$ & $-6,4873^{* \star *}$ & $-5,5214^{\star \star \star}$ \\
\hline LPOB & $-3,3206^{\star \star}$ & $-4,3144^{\star \star}$ & $3,2382^{\star * *}$ & $-3,2008^{\star *}$ & $-3,0948^{\star}$ & $-3,1878^{\star \star \star}$ \\
\hline
\end{tabular}




\begin{tabular}{|c|c|c|c|c|c|c|}
\hline LTC & $\begin{array}{c}- \\
3,8261^{\star \star \star}\end{array}$ & $-4,2875^{\star \star}$ & $-3,1632$ & $-3,9026^{\star \star \star}$ & $-4,3314^{\star \star}$ & $-2,5938^{\star \star}$ \\
\hline LPRP & $\begin{array}{c}-3,3782^{\star \star} \\
-\end{array}$ & $\begin{array}{c}- \\
4,2229 * \star \star\end{array}$ & $4,3935^{\star \star \star}$ & $-4,2823^{\star \star \star}$ & $-4,1165^{\star \star \star}$ & $-4,3154^{\star \star \star}$ \\
\hline LTI & $4,7068^{\star \star \star}$ & $-3,9923^{\star *}$ & $5,2687^{\star \star \star}$ & $-7,2307^{\star \star \star}$ & $-7,6570 * \star \star$ & $-6,0958 * \star \star$ \\
\hline
\end{tabular}

ADF: Augmented Dickey Fuller; PP: Phillips Perron.

Los rezagos para el ADF fueron predeterminados por el E-Views 6.0 (Max. 5) bajo Criterio Akaike.

I: Intercepto; II: Intercepto y Tendencia; III: Sin variable exógena.

Hipótesis Nula para ADF: "La serie tiene raíz unitaria"

Regla de decisión: si $p>\alpha$ no se rechaza la Hipótesis Nula. Si $p<\alpha$ se rechaza. $\alpha=0,1 ; 0,05$ y 0,01 para los niveles $10 \%, 5 \%$ y $1 \%$ respectivamente. *, ** $y{ }^{* * *}$ denotan rechazo de la Hipótesis Nula al $10 \%$, $5 \%$ y $1 \%$

\section{Anexo 2. Test de Cointegración de Engle-Granger}

Ecuación de Regresión Estimada (variables I(1))

Dependent Variable: LPOB

Method: Least Squares

Date: 05/13/09 Time: 19:17

Sample: 19752000

Included observations: 26

\begin{tabular}{ccccc}
\hline \hline & Coefficient & Std. Error & t-Statistic & Prob. \\
\hline \hline C & 4.850699 & 0.181831 & 26.67693 & 0.0000 \\
TI & -0.036154 & 0.004823 & -7.496979 & 0.0000 \\
PRP & -0.019402 & 0.006365 & -3.048446 & 0.0059 \\
TC & 0.000279 & 0.000196 & 1.419867 & 0.1697 \\
\hline \hline R-squared & 0.805821 & Mean dependent var & 3.799452 \\
Adjusted R-squared & 0.779342 & S.D. dependent var & 0.395406 \\
S.E. of regression & 0.185739 & Akaike info criterion & -0.388310 \\
Sum squared resid & 0.758978 & Schwarz criterion & -0.194756 \\
Log likelihood & 9.048026 & Hannan-Quinn criter. & -0.332573 \\
F-statistic & 30.43252 & Durbin-Watson stat & 1.564318 \\
Prob(F-statistic) & 0.000000 & & \\
\hline \hline
\end{tabular}


Test de Raíz Unitaria de los Residuos: La Regresión es Cointegrante

Null Hypothesis: RESIDUOS has a unit root

Exogenous: None

Lag Length: 0 (Automatic based on SIC, MAXLAG=5)

\begin{tabular}{cccc}
\hline \hline & t-Statistic & Prob.* \\
\hline \hline Augmented Dickey-Fuller test statistic & -4.217427 & 0.0002 \\
\hline Test critical values: & $1 \%$ level & -2.660720 & \\
& $5 \%$ level & -1.955020 & \\
& $10 \%$ level & -1.609070 & \\
\hline \hline
\end{tabular}

*MacKinnon(1996) one-sided p-values.

Anexo 3. Test de Cointegración de Soren-Johansen

Sample (adjusted): 19772000

Included observations: 24 after adjustments

Trend assumption: Linear deterministic trend (restricted)

Series: LPOB TI PRP TC

Lags interval (in first differences): 1 to 1

Unrestricted Cointegration Rank Test (Trace)

\begin{tabular}{ccccc}
\hline \hline $\begin{array}{c}\text { Hypothesized } \\
\text { No. of CE(s) }\end{array}$ & Eigenvalue & $\begin{array}{c}\text { Trace } \\
\text { Statistic }\end{array}$ & $\begin{array}{c}0.05 \\
\text { Critical Value }\end{array}$ & Prob.** \\
\hline \hline None * & 0.743246 & 78.85955 & 63.87610 & 0.0017 \\
At most $1 *$ & 0.589371 & 46.22823 & 42.91525 & 0.0225 \\
At most 2 & 0.509780 & 24.86664 & 25.87211 & 0.0663 \\
At most 3 & 0.276177 & 7.757016 & 12.51798 & 0.2722 \\
\hline \hline
\end{tabular}

Trace test indicates 2 cointegrating eqn(s) at the 0.05 level

* denotes rejection of the hypothesis at the 0.05 level

**MacKinnon-Haug-Michelis (1999) p-values 


\section{Apéndice A. Metodología}

La metodología utilizada para el análisis de los datos se basó en la estimación de un modelo de regresión múltiple, el cual permitió estudiar la relación cuantitativa estocástica entre la variable de interés considerada del tipo dependiente y un conjunto de variables explicativas o dependientes. En términos matriciales se tiene:

$$
\vec{Y}=X \vec{\alpha}+\vec{\varepsilon}
$$

donde $\vec{Y}$ es el vector de la variable respuesta o dependiente, $X$ es la matriz del diseño de las variables regresivas; $\vec{\alpha}$ es el vector de los parámetros del modelo, y $\vec{\varepsilon}$ es el vector de las perturbaciones aleatorias.

Es conveniente acotar que las hipótesis establecidas en la investigación reposan sobre un modelo planteado por los autores (ecuación (5)), las cuales fueron contrastadas posteriormente y explicadas con detalle en la presentación de resultados empíricos, una vez estimado el mismo. Partiendo del supuesto de un incremento proporcional de los niveles de pobreza total con respecto al crecimiento poblacional de una economía determinada, haciendo uso de la fórmula financiera del capital final a interés $\operatorname{simple}^{17}$, se tiene la siguiente expresión:

$$
P O B_{(t)}=P O B_{(t-1)}(1+n)
$$

donde $P O B_{(t)}$ representa la pobreza total en el momento $t$; $P O B_{(t-1)}$ corresponde a los niveles de la misma en el momento $t-1 ; n$ la tasa de crecimiento poblacional y $(1+n)$ el factor de crecimiento de la pobreza.

La expresión anterior restringe significativamente al modelo en el sentido de que solamente toma en cuenta la tasa de crecimiento poblacional y se sustenta en una función lineal. La literatura revisada da indicios de posibles determinantes que pueden aumentar o disminuir los niveles de pobreza total en un tiempo determinado bajo ciertas condiciones. Tomando en cuenta este fundamento, y basándose en la fórmula financiera de capital final a interés compuesto $^{18}$, se asume el componente $\lambda$ como el factor exponencial de crecimiento de la pobreza total, con $\lambda \in(-\infty,+\infty)$. Incluyendo éste último en (2) se tiene:

$$
P O B_{t}=P O B_{(t-1)}(1+n)^{\lambda}
$$

considerando que $0<n<1$ se asume $1<(1+n)<2$. Para efectos de simplificar el modelo, $n$ se considera constante en el tiempo y que $P O B_{(t-1)}$ se

\footnotetext{
${ }^{17} \mathrm{La}$ fórmula a la que se hace mención es la que indica que: $\mathrm{K}_{\mathrm{f}}=\mathrm{K}_{0}(1+\mathrm{i})$; donde $\mathrm{K}_{\mathrm{f}}$ es el capital final; $K_{0}$ es el capital inicial e i es el interés que gana el capital.

${ }^{18} \mathrm{La}$ fórmula de interés compuesto indica que $\mathrm{K}_{\mathrm{f}}=\mathrm{K}_{0}(1+\mathrm{i})^{\mathrm{t}}$; donde t es el tiempo de colocación del capital inicial $\left(\mathrm{K}_{0}\right)$ a un determinado interés (i), lo cual finalizado el período de colocación arroja un capital final $\left(\mathrm{K}_{\mathrm{f}}\right)$.
} 
comporta constante en el momento $t$, lo cual implica que $P O B_{(t)}$ dependerá directamente de $\lambda$, siendo éste el factor que tiende a aumentar o disminuir los niveles de pobreza total en un tiempo determinado. Por otro lado, considérese la ecuación:

$$
\lambda^{*}=\lambda[1-(\delta+\psi+\beta)]
$$

donde $\lambda^{*}$ representa el crecimiento exponencial afectado por $\delta$ el precio de realización del petróleo o barril petrolero, $\psi$ el tipo de cambio promedio y $\beta$ la tasa de inversión, suponiendo que $\delta, \psi$ y $\beta$ se encuentran normalmente distribuidas con media $\bar{\delta}, \bar{\psi}, \bar{\beta}$. En este orden de ideas, la expresión (4) implica que el factor de crecimiento exponencial bajo tales determinantes será igual a $\lambda$ siempre y cuando $(\delta+\psi+\beta)=0$. Por otra parte, si el impacto es positivo ocasionará que los determinantes propiamente dichos representen una disminución del factor de crecimiento exponencial de los niveles de pobreza total observados y por lo tanto $\lambda^{*}<\lambda$; sin embargo, si el impacto es negativo ocurre que $\lambda^{*}>\lambda$

Por otra parte, téngase en cuenta dos economías, con ${ }^{P O B_{(t-1)}}$ y $\mathrm{n}$ iguales, la primera con el factor de crecimiento exponencial de los niveles de pobreza total no afectado por los determinantes objeto de estudio (véase ecuación (2)) y la otra sí afectada. Ésta última quedaría representada por la ecuación:

$$
P O B_{(t)}=P O B_{(t-1)}(1+n)^{(\lambda-\lambda \delta-\lambda \psi-\lambda \beta)}
$$

Igualando las ecuaciones (2) y (5) para asumir que $P O B_{(t)}$ es igual que en ambas economías y tomando logaritmos naturales para linealizar la función:

$$
\ln P O B_{(t-1)}+\lambda \ln (1+n)=\ln P O B_{(t-1)}+(\lambda-\lambda \delta-\lambda \psi-\lambda \beta) \ln (1+n)
$$

Reordenando y eliminando términos comunes en (6) se obtiene:

$$
\lambda=\left[\lambda-\lambda \delta-\lambda \psi-\lambda \beta=\lambda_{0}\right]
$$

donde $\lambda_{0}$ puede ser considerado el valor crítico con el cual $\lambda=\lambda^{*}$, por lo tanto, la pobreza total en ambas economías son similares.

Haciendo uso de la igualdad en (7) y tomando derivadas parciales, se evaluaron matemáticamente el efecto que ejerce cada uno de los determinantes propiamente dichos sobre el factor de crecimiento exponencial de la pobreza, dando origen a las siguientes expresiones:

$$
\begin{aligned}
& \frac{d \lambda_{0}}{d \delta}=-\lambda<0 \\
& \frac{d \lambda_{0}}{d \psi}=-\lambda<0
\end{aligned}
$$




$$
\frac{d \lambda_{0}}{d \beta}=-\lambda<0
$$

La ecuación (8) indica que un shock positivo en los precios de realización del petróleo, es decir, un aumento en el precio unitario del barril, conlleva a una disminución en los niveles de pobreza total debido a la disminución del factor exponencial de crecimiento de la pobreza total. Por otra parte, un shock positivo en el tipo de cambio, véase ecuación (9), el cual puede verse reflejado en una revaluación o fortalecimiento de la moneda local de una economía en particular frente a la de otros países, afecta negativamente al factor exponencial de crecimiento de la pobreza total lo cual hace que $P O B_{(t)}$ disminuya en un tiempo determinado. Por último, la ecuación (10) implica que, ante la presencia de un shock positivo en la tasa de inversión, el exponencial se reduce por lo cual, se espera una disminución de los niveles de la pobreza total en la economía de referencia.

En concordancia con lo anterior, se establecieron un total de tres (3) hipótesis de acuerdo a las expresiones (8), (9) y (10), las cuales se analizaron y contrastaron una por una; es por ello que el análisis económico-estadístico fue fundamental, a efectos de responder a los planteamientos teóricos antes presentados. 